\title{
MIl partial tandem duplication induces aberrant Hox expression in vivo via specific epigenetic alterations
}

\author{
Adrienne M. Dorrance, ${ }^{1,2}$ Shujun Liu, ${ }^{1}$ Weifeng Yuan, ${ }^{1}$ Brian Becknell, ${ }^{1,3}$ \\ Kristy J. Arnoczky, ${ }^{3}$ Martin Guimond, ${ }^{1}$ Matthew P. Strout, ${ }^{1}$ Lan Feng, ${ }^{1}$ Tatsuya Nakamura, ${ }^{4}$ \\ Li Yu, ${ }^{1}$ Laura J. Rush,, ${ }^{2,5}$ Michael Weinstein, ${ }^{6}$ Gustavo Leone,, ${ }^{4,5}$ Lizhao Wu, ${ }^{4}$ \\ Amy Ferketich,5,7 Susan P. Whitman, 1,5 Guido Marcucci,1,5 and Michael A. Caligiuri1,2,3,4,5 \\ 1Department of Internal Medicine, Division of Hematology and Oncology, ${ }^{2}$ Department of Veterinary Biosciences, \\ ${ }^{3}$ Integrated Biomedical Science Graduate Program, ${ }^{4}$ Department of Molecular Virology, Immunology and Medical Genetics, \\ ${ }^{5}$ Comprehensive Cancer Center, ${ }^{6}$ Department of Molecular Genetics, and ${ }^{7}$ Division of Biometrics, The Ohio State University, Columbus, Ohio, USA
}

\begin{abstract}
We previously identified a rearrangement of mixed-lineage lenkemia (MLL) gene (also known as $A L L-1, H R X$, and HTRX1), consisting of an in-frame partial tandem duplication (PTD) of exons 5 through 11 in the absence of a partner gene, occurring in approximately $4 \%-7 \%$ of patients with acute myeloid leukemia (AML) and normal cytogenetics, and associated with a poor prognosis. The mechanism by which the MLL PTD contributes to aberrant hematopoiesis and/or leukemia is unknown. To examine this, we generated a mouse knockin model in which exons 5 through 11 of the murine $M l l$ gene were targeted to intron 4 of the endogenous $M l l$ locus. $M l^{P T D / W T}$ mice exhibit an alteration in the boundaries of normal bomeobox $(\mathrm{Hox})$ gene expression during embryogenesis, resulting in axial skeletal defects and increased numbers of hematopoietic progenitor cells. $M l l^{P T D} / W_{T}$ mice overexpress Hoxa7, Hoxa9 , and Hoxa10 in spleen, BM, and blood. An increase in histone $\mathrm{H3} / \mathrm{H} 4$ acetylation and histone $\mathrm{H} 3$ lysine 4 (Lys4) methylation within the Hoxa7 and Hoxa9 promoters provides an epigenetic mechanism by which this overexpression occurs in vivo and an etiologic role for $M L L$ PTD gain of function in the genesis of AML.
\end{abstract}

\section{Introduction}

Mixed-lineage leukemia ( $M L L$, also known as $A L L-1, H R X$, or HTRX1) is the human homolog of Drosophila Trx and is a maintenance factor for the homeobox (Hox) group of proteins, which play an important role in specifying cell fate during development and hematopoiesis (1). Approximately $4 \%$ of patients with de novo acute myeloid leukemia (AML) have balanced translocations or insertions that result in fusion of $M L L$ at chromosome 11q23 with 1 of over 40 functionally divergent genes. Although a central mechanism responsible for malignant transformation as a result of these chromosomal translocations involving 11q23 is lacking, the $\mathrm{N}$ terminus of MLL, which contains the AT-hook DNA-binding motif and a region homologous to DNA methyltransferase, is always retained in the fusion protein while the $\mathrm{C}$ terminus, which contains the activation and SET domains, is always replaced by the fusion partner (2).

We discovered a rearrangement of $M L L$ in AML patients whereby $M L L$ is not fused with a partner gene but rather is consistently elongated with an in-frame partial tandem duplication (PTD) of exons 11-5 or 12-5 (former exon designations were 6-2 and 8-2) (3). In contrast with the myriad of $M L L$ fusions in AML that result from chromosomal translocations, AML blasts with the MLL PTD retain the protein's $C$ terminus, which contains the

Nonstandard abbreviations used: AML, acute myeloid leukemia; BFU-E, burstforming unit-erythroid; ChIP, chromatin IP; GEMM-CFU, granulocyte, erythroid, macrophage, megakaryocyte-CFU; Hox, homeobox; Lys4, lysine 4; MLL, mixed-lineage leukemia; PTD, partial tandem duplication.

Conflict of interest: The authors have declared that no conflict of interest exists. Citation for this article: J. Clin. Invest. 116:2707-2716 (2006). doi:10.1172/JCI25546 activation and SET domains and partially duplicates a region within the $\mathrm{N}$ terminus containing the AT-hook DNA-binding and repression domains (2). Approximately 4\%-7\% of AML patients with normal cytogenetics harbor the MLL PTD and carry an especially poor prognosis $(4,5)$.

HoxA genes are frequently overexpressed in leukemia (6). The mechanism by which the MLL PTD contributes to aberrant hematopoiesis and/or leukemogenesis is currently unknown, but given MLL's role as a maintenance factor for Hox proteins, 1 mechanism could involve deregulation of Hox gene expression. In in vitro model systems, WT MLL regulates certain $H O X$ gene expression via epigenetic modification at cis-regulatory regions, i.e., histone $\mathrm{H} 3 / \mathrm{H} 4$ acetylation and $\mathrm{H} 3$ lysine 4 (Lys4) methylation, the latter of which requires MLL's C terminus SET domain $(7,8)$. When the $M L L-A F 9$ fusion gene (the result of one of the most common MLL rearrangements) is transduced into $\mathrm{Mll}^{-/-}$cells, Hoxa9 is overexpressed by an unknown mechanism that does not require binding of the MLL-AF9 fusion directly to the Hoxa9 promoter (8). However, in another in vitro study, transduction of the MLL-eleven nineteen leukemia and the MLLFK506-binding protein fusions into MllWT/WT cells increased Hoxa 9 expression via $\mathrm{H} 3$ acetylation but without increased H3 Lys4 methylation (9). Likewise, when the Mll-AF9 fusion was knocked in as an endogenous allele, mice showed Hoxa9 overexpression and developed AML, but no epigenetic or other alteration could account for this overexpression $(10,11)$. This suggests distinct mechanisms for induction of HOX genes by WT MLL and fusions of MLL resulting from balanced chromosomal translocations.

Taspase 1 cleaves the approximately 430-kDa MLL protein into 2 smaller components, and this cleavage has been shown to 


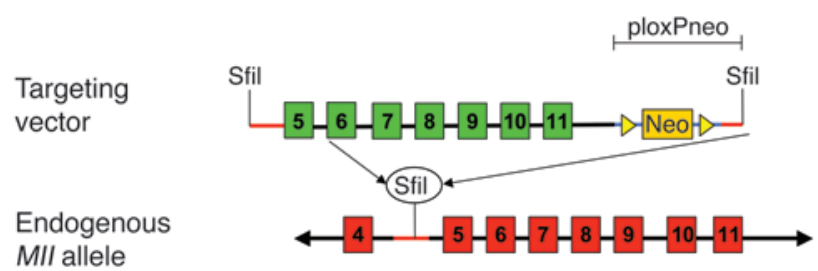

Knocked-in MII PTDallele

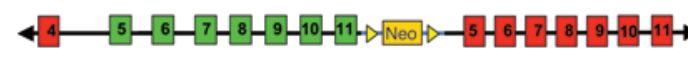

Cre-Recombinase

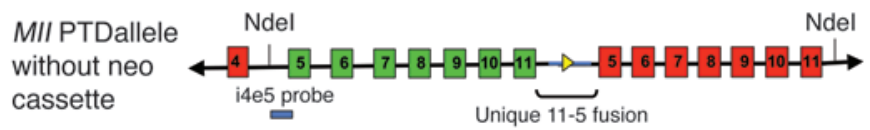

be essential for normal MLL function and regulation. Absence or downregulation of taspase 1 leads to absence or decreases in transcription of normal downstream targets of MLL, such as the Hox genes (12). In virtually all cases involving reciprocal $M L L$ translocations, the consensus sequences for taspase 1 are lost and replaced by the fusion partner, resulting in one of the only known common features among all the reciprocal translocations involving MLL. However, the MLL PTD differs from translocations in that the consensus sequences for taspase 1 are retained and the MLL PTD protein is cleaved (13). How these MLL mutations function with or without taspase 1 cleavage is currently unsolved but highlights another important difference between MLL fusions and the MLL PTD.

In this study, we created a mouse expressing the Mll PTD off of the endogenous $\mathrm{Mll}$ promoter and examined the molecular basis for its phenotype. We show that this mouse has overexpression of certain HoxA genes, axial skeletal defects, and aberrant hematopoiesis, all compared with age- and sex-matched littermate controls. We also show that the Mll PTD is associated with increased histone $\mathrm{H} 3 / \mathrm{H} 4$ acetylation and methylation of H3 Lys 4 at cis-regulatory HoxA sequences, providing what we believe to be the first in vivo evidence for a mechanism by which an $M L L$ gene rearrangement can directly alter HoxA gene expression.

\section{Results}

$M I l^{P T D / W T}$ mice are viable and express the fusion transcript. To study the in vivo consequences of the Mll PTD and to more closely model the monoallelic involvement seen in human AML with the MLL PTD (14), we employed homologous recombination in ES cells to introduce a genomic fragment containing exons 5 through 11 into intron 4 of $M l l$ (Figure 1). Using $2 \mathrm{Mll}^{P T D / W T}(+)$ ES clones, we obtained 11 mice with germline transmission of the Mll PTD and backcrossed these mice with C57BL/6J mice to obtain $M l l^{P T D / W T}$ mice on a pure (congenic) C57BL/6J background. Southern blotting (Figure 2A) and DNA PCR (not shown) verified the presence of the $M l l^{P T D / W T}$ genotype. Southern blotting on 1-day-old pups from 5 matings also confirmed that $M l l^{P T D / P T D}$ homozygous mice are embryonic lethal (Table 1). Sequencing of the RT-PCR product from $M I l^{P T D / W T}$ mice demonstrated a correctly spliced, in-frame fusion transcript confirming the precise targeting of exons 5 through 11 within intron 4 of the Mll locus (Figure 2B).

\section{Figure 1}

Generation of $M I I^{P T D / W T}$ mice. The backbone of the targeting vector is ploxPneo 1 and contains a genomic clone spanning MII intron 4 through intron 11. Linearization of the vector at a unique Sfil site within intron 4 allows for recombination at the germline intron 4 of MII. A plasmid vector expressing Cre recombinase from a CMV promoter ( $\mathrm{pCre}$ ) was used to excise the neo cassette.
Absolute quantification of WT and PTD transcripts in MllPTD/WT mice using real-time RT-PCR. Real-time primers and probes were designed and optimized, amplifying the unique 11-5 Mll PTD fusion present only in $M l l^{P T D / W T}$ mice as well as the 14-15 Mll exon-exon junction located outside the duplicated region and common to both the $M l l$ PTD allele and the Mll WT allele. Using these probes in an absolute quantification assay (13), it was determined that the Mll PTD and Mll WT alleles were both expressed at low, near equivalent levels in the hematopoietic tissues of the $M l l^{P T D} / W T$ mice (Figure 3 and Table 2). Protein confirmation of this equal yet low level of gene expression by Western blot was not technically possible.

Phenotypic abnormalities of $M l l^{P T D / W T}$ mice. Examination of $M l l^{P T D / W T}$ mice revealed skeletal abnormalities compared with $M l l^{W T / W T}$ ageand sex-matched littermate controls. Radiographic examination
A

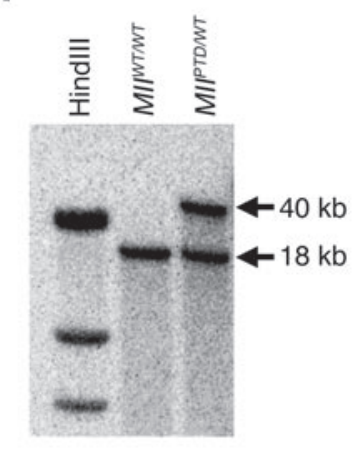

B
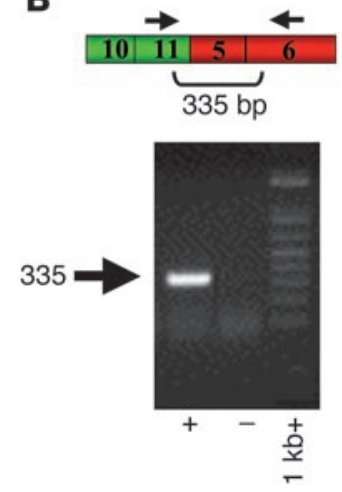

Figure 2

Germline transmission and verification of correct targeting in mice heterozygous for the MII PTD. (A) Southern blot analysis using high molecular-weight DNA from spleens, digested with Ndel and hybridized to a probe that spans intron 4 and exon 5 of MII (i4e5 in Figure 1). This generates a single WT band at $18 \mathrm{~kb}$ in MIIWT/WT mice, 2 bands representing $1 \mathrm{WT}$ allele at $18 \mathrm{~kb}$, and a band at $40 \mathrm{~kb}$ representing the rearranged allele in MIIPTD/WT mice. (B) The MII PTD fusion transcript was amplified using an upstream primer from MII exon 11 and a downstream primer from MII exon 6 amplifying a single 335-bp unique fusion transcript in the MIIPTD/WT mouse (+) and is absent in the sample from the MIIWT/WT mouse (-). Sequencing of these PCR products from the $M I I P T D / W T$ mice verified the presence of the exon 11-exon 5 fusion site. The 1-kb+ ladder was used to determine amplification size. 
Table 1

Assessment of Mendelian ratios for the MII PTD allele

$\begin{array}{lcc}\text { Matings } & \begin{array}{c}\text { Matings } \\ \text { (no.) }\end{array} & \begin{array}{c}\text { Total MIIWTWT offspring } \\ \text { (expected freq/actual freq) }\end{array} \\ & & \end{array}$

$\begin{array}{lll}\text { MIIPTDNT } \times \text { MIIPTDNT } & 5 & 8(25: 30)\end{array}$
Total MIIPTD/WT offspring

(expected freq/actual freq)

$19(50: 70)$
Total MIIPTD/PTD offspring

(expected freq/actual freq)

$0(25: 0)$

${ }^{A} \mathrm{All}$ ratios in parentheses indicate percentages. Freq, frequency.

of $M l l^{P T D / W T}$ mice less than 1 year of age identified axial skeleton malformations, including a rudimentary or missing thirteenth rib, indicative of a $\mathrm{T} 13 \rightarrow \mathrm{L} 1$ transformation ( $70 \%$ penetrance, $n=13$ ), and an $\mathrm{S} 1$ vertebral duplication, indicative of an $\mathrm{S} 2 \rightarrow \mathrm{S} 1$ transformation $(63 \%, n=11)$ (Figure 4, B and C).

Given the WT Mll's role in maintaining Hox expression during development and the known association between HoxA expression, anteroposterior patterning, and posterior axis elongation, we examined Hoxa9 expression during embryogenesis. In situ hybridization performed on E12.5 MllPTD/WT embryos demonstrated a shifted expression boundary of Hoxa9 within the paraxial mesoderm compared with $M l l^{W T / W T}$ littermate controls (Figure 5).

Altered hematopoiesis in MllPTD/WT mice is associated with aberrant HoxA expression. Lineage-specific CFU assays were performed for erythroid (measured by erythropoietic burst formation [BFU-E]), myeloid, (measured by GM-CFU), and mixed (measured by granulocyte, erythroid, macrophage, megakaryocyte-CFU [GEMM$\mathrm{CFU}]$ ) progenitor populations, using both $M l l^{P T D / W T}$ and $M l l^{W T / W T}$ splenocytes. The CFU number for each progenitor type was significantly increased in $M l^{P T D / W T}$ mice compared with age- and sexmatched $M l l^{W T / W T}$ littermate controls (Figure 6A). A 5- to 20-fold increase in $M l l^{P T D / W T}$ spleen cells expressing the erythroid marker Ter119 was also noted, consistent with the observed increase in erythroid progenitor populations as measured by BFU-E (Figure 6, $B$ and $C)$. However, extensive phenotypic analyses performed as previously described (15) on blood, spleen, and BM did not reveal any other differences in $M l^{P T D / W T}$ mice compared with age- and sex-matched $M l^{W T T W T}$ littermate controls (see representative flow cytometric analyses, Supplemental Figure 1; available online with this article; doi:10.1172/JCI25546DS1). Primary CFUs were then harvested and used in 2 parallel secondary assays. In replating CFU assays, the total number of colonies per plate was not significantly different between $M l l^{P T D / W T}$ and $M l l^{W T / W T}$ splenocytes, yet the size of the MIPTD/WT colonies was markedly increased over both $M l W W T / W T$ colonies and those derived from MllAfg/WT mice (Figure 6, D-F). Secondary colonies from $M l l^{P T D / W T}$ mice were able to form tertiary and quaternary colonies while those from $M l l^{W T / W T}$ and $M l l^{A \rho P / W T}$ mice were not (data not shown). The increase in size per $M I P T D / W T$ colony suggested an increase in progenitor cell proliferation compared with $M l L^{W T / W T}$ progenitor cells. Nonetheless, absolute in vivo cell counts in bone marrow, spleen, and blood were not significantly different between $M l^{P T D / W T}$ and $M l l^{W T / W T}$ mice, suggesting that enhanced proliferation in the $M I l^{P T D / W T}$ progenitor cells was accompanied by increased cell turnover. To verify this, primary $\mathrm{CFU}$ colonies were harvested and maintained in liquid cultures supplemented with SCF, IL-3, and IL-6 for an additional 18 days, followed by cell counts, measurement of BrdU uptake for quantification of proliferation, and assessment of apoptosis by annexin $\mathrm{V}$ and propidium iodide staining. The $M I l^{P T D / W T}$ hematopoietic progenitor cells did have a 2-fold great- er fraction of cells incorporating BrdU compared with $M l l^{W T / W T}$ progenitor cells (Figure 6, G-H) but also showed a substantial amount (50\%) of apoptosis (Figure 6I) such that absolute cell counts over this period of time were only modestly increased from the initiation of culture (Table 3). This near maintenance of homeostasis could also explain the normal cell counts observed in bone marrow, spleen, and blood of $M l l^{P T D / W T}$ mice in vivo. The lack of viable $M I l^{W T / W T}$ cells at day 18 in repeated assays (Table 3) precluded our ability to obtain statistically meaningful comparative data in repeated cultures. In contrast, $M l l^{P T D / W T}$ progenitor cells were sustained in liquid culture for more than 4 months.

Increased expression of HoxA genes in MIlPTD/WT mice. To establish a possible cause for the aberrant increases in both the number of primary colonies and increased proliferative capacity of replated progenitors from $M l^{P T D / W T}$ mice, we quantified expression of Hoxa7, Hoxa9, and Hoxa10 within hematopoietic tissues. We found consistent and significant overexpression of each HoxA gene in BM, spleen, and blood when compared with their expression in matched MllWT/WT littermate controls (Figure 7, A-C). Expression of Hoxa1, a HoxA family member that is not regulated by $\mathrm{Mll}$, was unaltered in any of the hematopoietic tissues examined while Hoxc8, a HoxC family member that has been shown to be positively regulated by WT MLL (8), was also not upregulated in any of the tissues examined (not shown).

The lack of significant differences in cellular populations of blood, spleen, and BM between $M l l^{P T D / W T}$ and $M l l^{W T / W T}$ mice as noted above suggested that the increased HoxA gene expression was due to an increase in the HoxA expression on a per cell basis. To confirm this, we sorted equal numbers of cells from BM of MllPTD/WT and MllWT/WT mice expressing different lineage markers: B220, CD3, CD11b, Gr-1, and Ter119. RNA was isolated from an equivalent number of cells and reverse transcribed into cDNA. Using real-time RT-PCR, Hoxa9
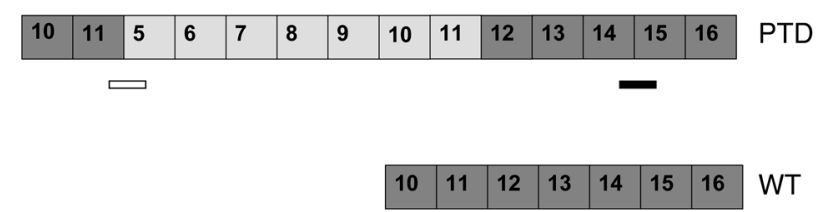

\section{Figure 3}

MII WT and MII PTD transcripts. Schematic demonstrating the realtime RT-PCR strategy for detection and absolute quantification of the MII WT and MII PTD transcripts in bone marrow and spleen of MII PTD mice. The predicted MII PTD and MII WT allele-derived transcripts are shown, with the tandemly duplicated exons 5-11 present in the PTD transcript denoted with light gray boxes. Shown below the transcripts are sites for PCR primers and fluorogenic probes designed to amplify either the unique exon 11-exon 5 fusion (open rectangle) or exons $14-15$, whose junction is common to the MII WT and MII PTD transcripts (filled rectangle). 
Table 2

Absolute quantitation of MIIPTD and MIIWT transcripts

\begin{tabular}{lcccc} 
Genotype & $\begin{array}{c}\text { Copy no. MII } \\
\text { PTD amplicon } \\
\text { (11-5 fusion) }\end{array}$ & $\begin{array}{c}\text { Copy no. } \\
\text { MII common amplicon } \\
(\mathbf{1 4 - 1 5} \text { exons) }\end{array}$ & Ratio & SD \\
MIIPTDNT & 367 & 920 & $2.5^{\mathrm{A}}$ & 0.3 \\
MIINTWT & - & 2070 & - & - \\
\hline
\end{tabular}

To determine whether the equivalent amount of transcript was being produced from each allele in the MIIPTD/WT tissues, the MII PTD unique fusion amplicon copy number was subtracted from the common amplicon (shared between the MII PTD and MII WT alleles) copy number. Alf both alleles were transcribed at equivalent rates, the common-tounique amplicon copy number ratio would be approximately $2: 1$ in a sample with the MIIPTD/WT genotype or approximately $1: 1$ if the MII WT allele was silenced (13). $n=3 /$ genotype.

was shown to be overexpressed in each of the $M l^{P T D / W T}$ cell subsets when compared with the identical MllWT/WT subset, consistent with an increase in HoxA gene expression on a per cell basis (Figure 7D).

Epigenetic alterations at HoxA gene promoters in $M l l^{P T D / W T}$ mice. As noted earlier, HoxA gene overexpression has been noted in both in vitro and in vivo models of the $M L L-A F 9$ fusion, but an in vivo mechanism to explain this upregulation in this or any $M L L$ rearrangement associated with leukemia has yet to be provided (11). Two groups have shown that WT MLL has intrinsic histone H3 Lys 4 methyltransferase activity within its $C$ terminus SET domain $(7,8)$, which is retained in the MLL PTD. To test whether epigenetic modifications within HoxA promoters were responsible for HoxA overexpression in $M l l^{P T D / W T}$ hematopoietic tissues, chromatin immunoprecipitation (ChIP) was performed within promoter sequences known to be important for maintaining HoxA transcription and spleen and BM were compared with similar tissues in $M l l^{W T / W T}$ mice. MllPTD/WT mice displayed an increase in histone $\mathrm{H} 3$ and $\mathrm{H} 4$ acetylation and $\mathrm{H} 3$ Lys 4 methylation with a corresponding decrease in H3 Lys9 methylation within the Hoxa9 (Figure 8, A and B) and Hoxa7 (not shown) promoters. These epigenetic modifications associated with gene transcription establish a mechanistic link between the $M l l^{P T D / W T}$ genotype and the associated overexpression of Mll targets Hoxa7 and Hoxa9. No such epigenetic changes were noted within the pertinent promoter region of Hoxa10.

We further assessed whether the increased histone modifications associated with gene activation were due to a gain of function of the Mll PTD and not the result of losing a WT Mll allele. H3/H4 acetylation and H3 Lys4 methylation at the Hoxa7 and Hoxa9 promoters were therefore measured using ChIP in combination with SYBR green real-time PCR in $M l^{P T D / W T}$ and $M l l^{W T /-} \mathrm{BM}$ and spleen and compared with similar measurements in $M l l^{W T / W T} \mathrm{BM}$ and spleen. Results show no significant difference in the histone modifications at these promoters when comparing $M l l^{W T /-}$ and $M l l^{W T / W T}$ tissues. In contrast, $M l l^{P T D / W T}$ tissues showed significantly increased $\mathrm{H} 3 / \mathrm{H} 4$ acetylation and $\mathrm{H} 3$ Lys 4 methylation at the Hoxa7 and Hoxa9 promoters when compared with these other 2 genotypes (Figure 8, C and D, and Table 4). These data indicate that the histone modifications associated with gene activation in the Mll ${ }^{P T D / W T}$ cells result from gainof-function mutation involving the PTD and not simply the loss of a WT Mll allele. It should also be noted that HoxA gene expression in $M l l^{P T D / W T}$ E17.5 fetal liver cells showed a 5- to 10-fold increase in Hoxa7, Hoxa9, and Hoxa10 gene expression compared with either MllWT/WT or $M l^{W T /}$ - fetal liver cells (data not shown). Finally, preliminary experiments comparing fetal liver cell HoxA gene expression obtained from $M l l^{P T D / W T}, M l l^{W T /-}$, and $M l l^{P T D /-}$ E17.5 embryos strongly support the conclusion that the Mll PTD confers a gain-of-function mutation (A. Dorrance and M. Caligiuri, unpublished observations).

Absence of leukemic transformation in Mll ${ }^{P T D / W T}$ mice highlights important requirement for second hit in the progression of lenkemia. Despite the aberrant hematopoiesis, the mice had not developed leukemia over 2 years of observation. Hoxa9 cooperates with overexpressed Meis1 in the induction of murine AML (16-18). We therefore quantified Meis1 transcript in affected hematopoietic tissues but found no evidence of overexpression (not shown). Likewise, we have noted that AML blasts from patients harboring the MLL PTD consistently lack expression of the WT MLL allele (13); however, hematopoietic tissues of $M l l^{P T D} / W T$ mice showed near equivalent levels of WT and PTD transcript (Figure 2C). Thus, it is possible that these and/or other additional molecular alterations are required for malignant transformation and can explain the aberrant hematopoiesis in the absence of leukemic transformation in $M l l^{P T D / W T}$ mice.

\section{Discussion}

In the current report, we describe a mouse engineered with $\mathrm{Mll}$ PTD expression driven by its endogenous promoter. The $M l l^{P T D / P T D}$ genotype appears embryonic lethal, and characterization of these
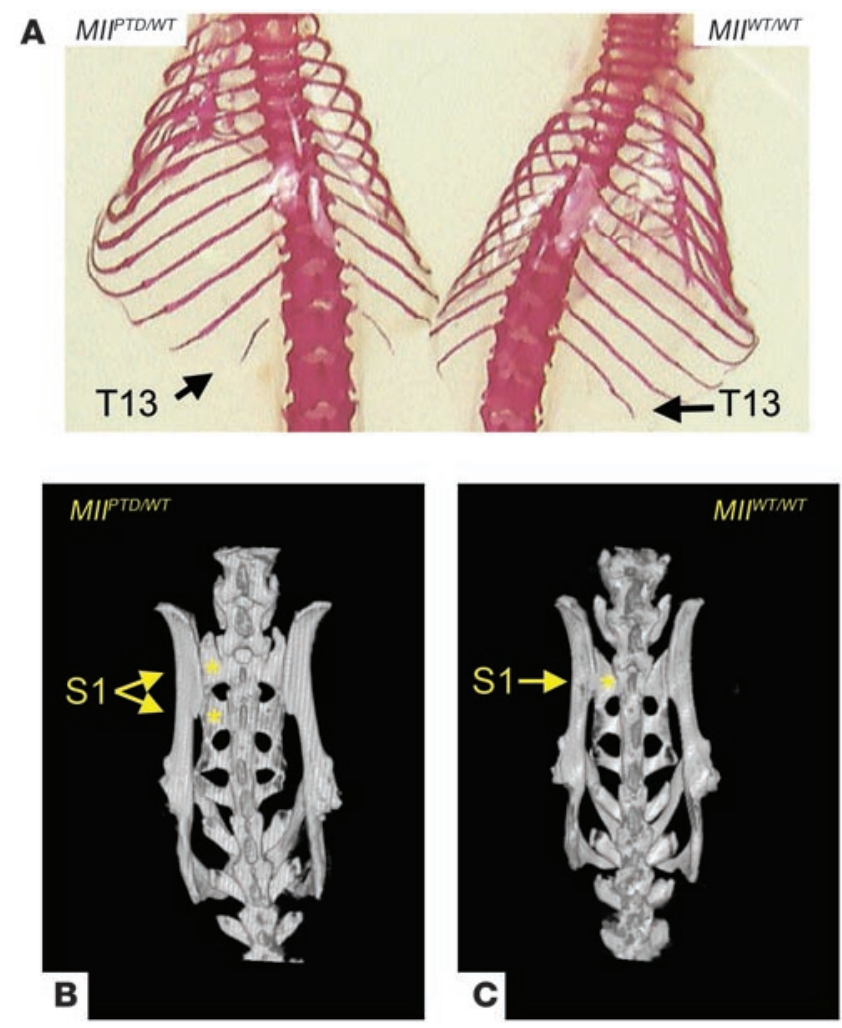

\section{Figure 4}

Skeletal analysis of MIIPTD/WT mice at 20 weeks of age. (A) Alizarin red staining of MIIPTD/WT (left) and MIIWT/WT (right) mice shows a rudimentary/missing thirteenth rib, indicative of a T13 $\rightarrow \mathrm{L} 1$ transformation (penetrance of $70 \%$ in MIIPTD/WT mice, $n=13$ ). MicroCT images of the sacral spine in a (B) MIIPTD/WT mouse and a (C) MIIWT/WT mouse in 3D. These images are viewed from the dorsal side and illustrate the duplication of the S1 vertebral body, indicative of an S2 $\rightarrow$ S1 transformation in the MIIPTD/WT mice (penetrance of $63 \%, n=11$ ). 
A

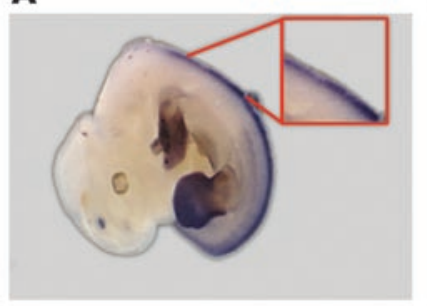

B

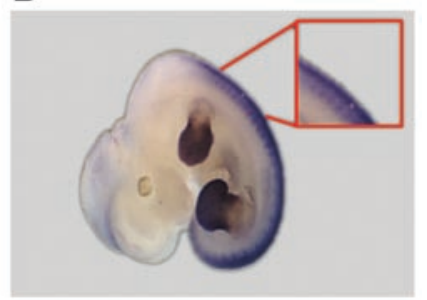

\section{Figure 5}

In situ hybridizations for Hoxa9 on E12.5 embryos. (A) MIIWT/WT and (B) MIIPTD/WT embryos hybridized with a digoxygenin-labeled probe for Hoxa9. Results demonstrate a notable ventral shift of the Hoxa9 expression boundary within the paraxial mesoderm of the MIIPTD/WT embryo compared with the MIIWT/WT littermate control (see inset for each figure). This figure represents 1 of 3 comparable hybridizations. embryos is ongoing. $M I l^{P T D / W T}$ mice are viable, are able to reproduce, and are without serious illness after 2 years of observation. However, $M I l^{P T D} / W T$ mice have a high penetrance of skeletal abnormalities that consist of a missing or rudimentary thirteenth rib and a duplication of the S1 vertebral body.

Hematopoietic abnormalities consist of significantly increased BFU-E, GM-CFU, and GEMM-CFU progenitors in cultures from $M I l^{P T D} / W T$ splenocytes when compared with age- and sex-matched $M l L^{W T / W T}$ littermate controls. Alterations in members of the homeo- box or Hox family of genes could reasonably explain these seemingly diverse phenotypic findings in the Mll PTD mice since certain Hox members are critical in axioskeletal and hematopoietic development and maintenance of Hox expression is positively regulated by WT Mll (1). The current report documents the increased expression of selected HoxA genes that is coincident with the Mll PTD and, for what we believe is the first time, provides a mechanism by which an $M l l$ defect that is associated with AML causes deregulation of HoxA expression in vivo.
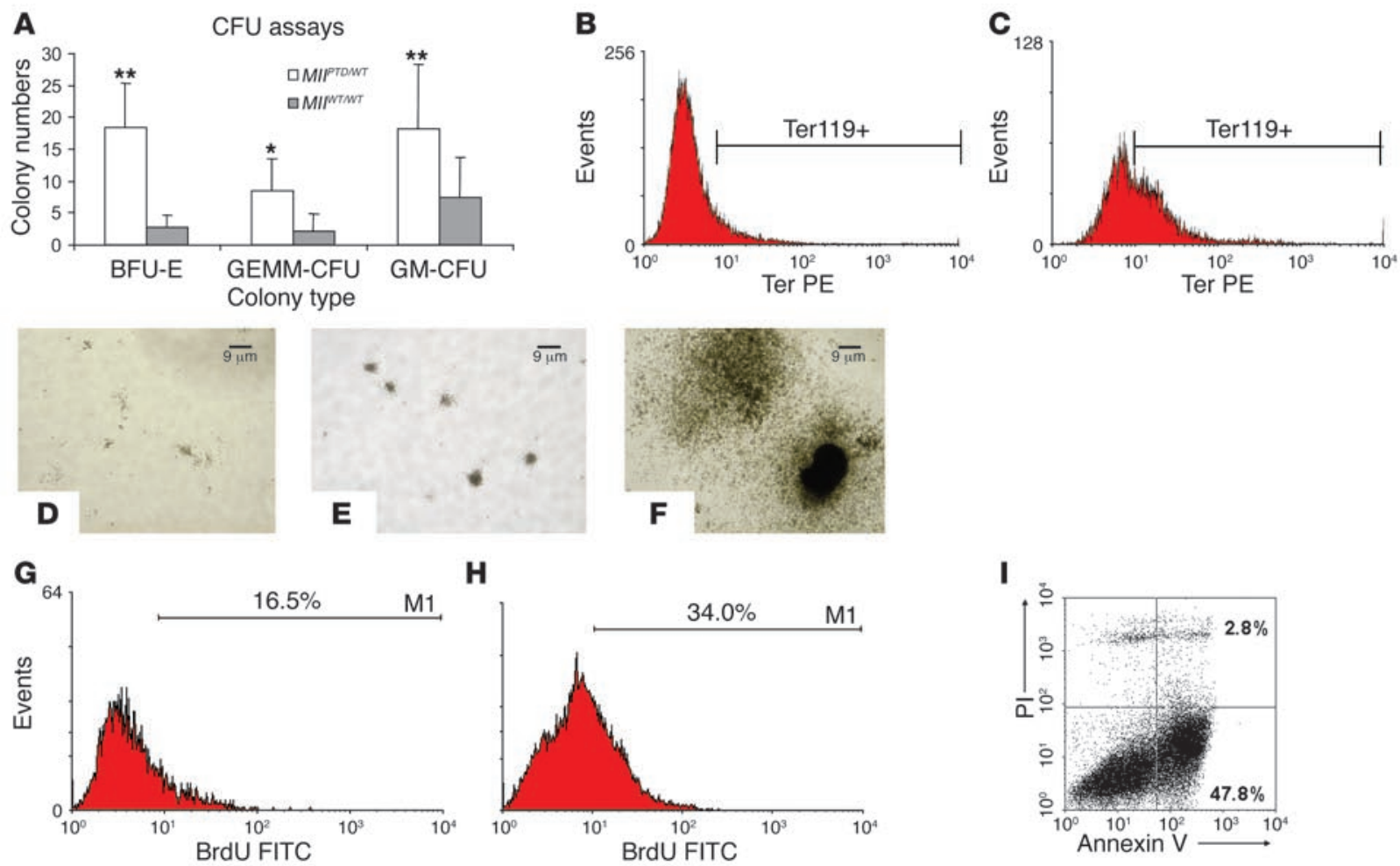

H
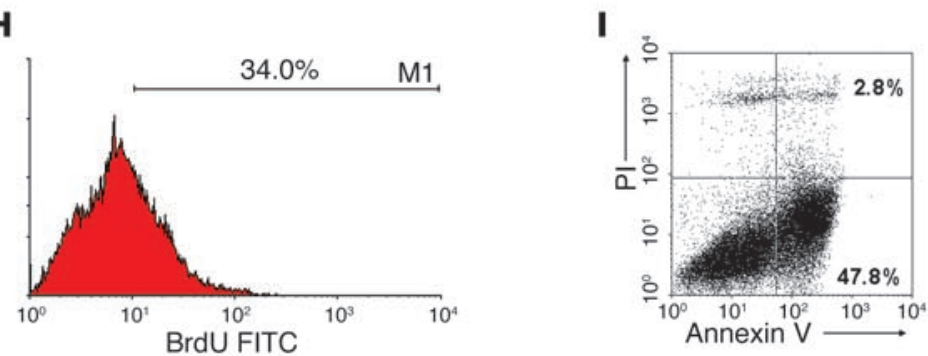

Figure 6

Evaluation of progenitor populations in MIIPTD/WT splenocytes compared with MIIWT/WT sex-matched littermate controls. (A) Results from CFU assays to assess progenitors of erythroid (BFU-E), granulocytic, erythroid, monocytic, megakaryocytic (GEMM-CFU), and granulocyte, macrophage (GM-CFU) lineages show significantly increased CFUs derived from MIIPTD/WT versus MIIWT/WT splenocytes. ${ }^{*} P=0.03 ;{ }^{* *} P=0.0025$. Error bars indicate SD. Splenocytes from (B) MIIWT/WT and (C) MIIPTD/WT mice ( $n=8$ mice per genotype) show increased surface density expression of the erythroid marker Ter119 in MIIPTD/WT mice. Ter PE, PE fluorochrome-conjugated Ter119. Secondary splenic colonies from (D) MIIWT/WT, (E) $M I / A f / W T$, and $(\mathbf{F}) M I I P T D / W T$ mice $(n=6$ mice per genotype, plated in duplicate). The numbers of secondary colonies per plate $(\sim 230)$ were similar between genotypes. The number of cells per colony was dramatically increased in MIIPTD/WT mice compared with MI/WT/WT or MIIAfg/WT mice. (G-I) Primary CFU progenitor cells harvested after 14 days and maintained in liquid cultures for 18 days, supplemented with SCF, IL-3, and IL-6, and in the presence of BrdU for the last 4 days. An anti-BrdU antibody gated on viable cells demonstrates a smaller fraction of $M I / W T / W T$ cells proliferating (G) compared with MIIPTD/WT cells $(\mathbf{H})$. Assessment of programmed cell death (I, upper right and lower right quadrants) revealed a sizeable fraction ( $50 \%)$ of $M I I^{P T D / W T}$ progenitors undergoing apoptosis during expansion. While a significant fraction of $M I I^{P T D / W T}$ cells were proliferating, approximately $50 \%$ were undergoing apoptosis. In 2 additional experiments, the MIIWT/WT cells had already died by this time point in culture (see Table 3), precluding a statistical comparison of these results in $\mathbf{G}-\mathbf{I}$. 
Table 3

Splenic progenitor cells grown in liquid culture

\begin{tabular}{lccc}
\hline Genotype & $\begin{array}{c}\text { Day 0 } \\
\text { (no. cells) }\end{array}$ & $\begin{array}{c}\text { Day 7 } \\
\text { (no. cells) }\end{array}$ & $\begin{array}{c}\text { Day 18 } \\
\text { (no. cells) }\end{array}$ \\
MIIWTWT & $2.2 \times 10^{6}$ & $1.2 \times 10^{5}$ & $1.3 \times 10^{4}(\downarrow)$ \\
MIIWTWT & $7.5 \times 10^{4}$ & All dead & All dead \\
MIINTWT & $9.9 \times 10^{5}$ & $6.9 \times 10^{5}$ & All dead \\
MIPTDWT & $6.2 \times 10^{6}$ & $1.3 \times 10^{6}$ & $7.5 \times 10^{6}(\uparrow)$ \\
MIIPTDWT & $3.7 \times 10^{6}$ & $1.2 \times 10^{6}$ & $1.5 \times 10^{7}(\uparrow)$ \\
MIPTDWT & $6.0 \times 10^{6}$ & $1.9 \times 10^{6}$ & $8.7 \times 10^{6}(\uparrow)$
\end{tabular}

ASignifies the start of liquid cultures with cells harvested from day 14 CFUs. Arrows indicate increase or decrease in number of cells at day 18 compared with day 7 .

Hox genes display spatiotemporal expression patterns that are colinear with respect to their location on the chromosome (19). Thus, for normal skeletal development to proceed, Hox genes must be expressed in the right cell type at the right time. Expression of these genes at incorrect times or locations leads to disruptions in normal Hox expression boundaries, resulting in altered cell fate and misspecification of segment identities (20-22). To determine whether the malformations of the axial skeletons of our MIlPTD/WT mice were associated with alterations in Hox expression boundaries, we performed whole-mount in situ hybridizations on E12.5 embryos and found the expression of Hoxa9 in the MlPTD/WT embryos extended laterally in the somitic mesoderm when compared with $M l l^{W T / W T}$ littermate controls. This finding is consistent with other reports that have implicated Hoxa9 as an important gene for lumbosacral patterning (23).

Restricted Hox expression is also vital for normal hematopoiesis, and deregulation in HoxA expression can result in aberrant hematopoiesis and malignant transformation (18, 24-30). Following the observations of progenitor cell abnormalities in $M l l l^{P T D / W T}$ spleen, we noted significant increases in Hoxa7, Hoxa9, and Hoxa10 transcripts in the spleen, $\mathrm{BM}$, and blood of $M l l^{P T D / W T}$ mice when compared with $M l l^{W T / W T}$ littermate controls and with $M l l^{W T /-}$ mice. However, Hoxa1, a HoxA family member not regulated by $\mathrm{Mll}$, showed no increase in its expression in tissues of the MlPTD/WT mice. Likewise, Hoxc8, a member of the HoxC paralogous group that has been shown to be a direct target of MLL, was not increased within these tissues, indicating the specific effect of the Mll PTD on these HoxA genes. Further, as these HoxA family members are positively regulated by WT Mll, their consistent and significant overexpression in the tissues of $M l l^{P T D / W T}$ mice also suggests that the Mll PTD was operating in a gain-of-function fashion.

WT MLL has been shown to directly and indirectly modify histones at the promoters of certain HOX genes, thereby maintaining gene transcription $(7,8)$. Histone modifications are associated with gene activation and repression (31-33). Histone acetylation is the predominant modification associated with gene activation as well as histone $\mathrm{H} 3$ Lys 4 methylation, while histone H3 Lys9 methylation corresponds with gene repression. Enzymes
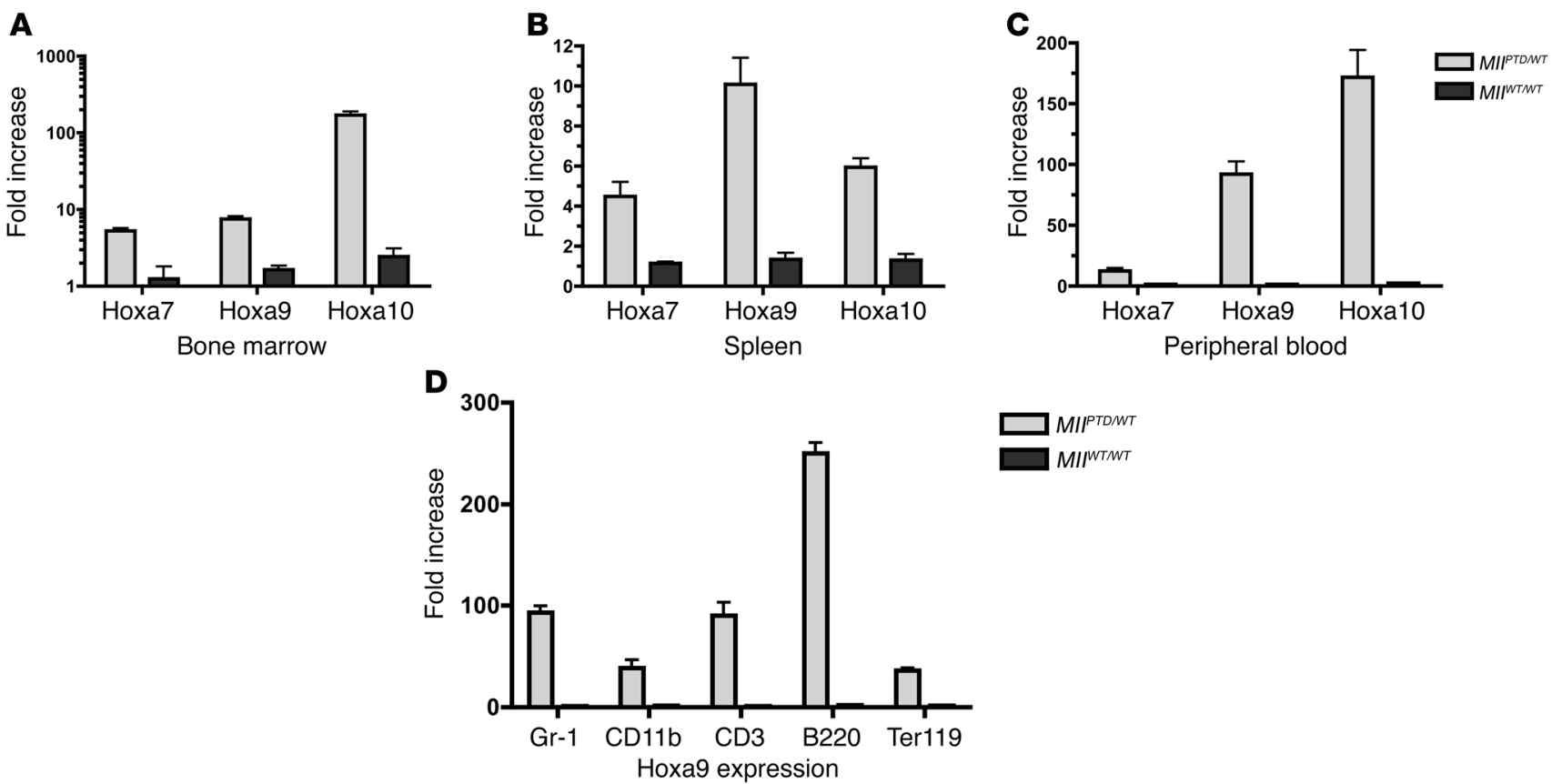

Figure 7

HoxA gene expression levels. (A-C) Quantification of Hoxa7, Hoxa9, and Hoxa10 expression in hematopoietic tissues of MIIWT/WT and MIIPTD/WT mice. Cells were isolated from (A) BM, (B) spleen, and (C) blood from both MI/WT/WT and MIIPTD/WT mice ( $n=10$ mice per genotype). RNA and then cDNA were prepared and quantified by real-time RT-PCR. MIIPTD/WT mice showed increased expression of each of these HoxA genes in all 3 tissues compared with sex-matched littermate MIITT/WT mice. (D) Equal numbers of each sorted BM population expressing Gr-1, CD11b, CD3, B220, or Ter119 from MIIPTD/WT mice and MIIWT/WT mice were processed for RNA and then cDNA. Absolute quantification of Hoxa9 expression was then determined by real-time RT-PCR and demonstrated that MIIPTD/WT cells have increased Hoxa9 expression within each population compared with the equivalent number of $M I / W T / W T$ cells, indicating that Hoxa9 transcript levels are overexpressed on a per cell basis (values shown represent mean of $n=3$ mice per genotype $\pm \mathrm{SD} ; P<0.03$ ). 
A

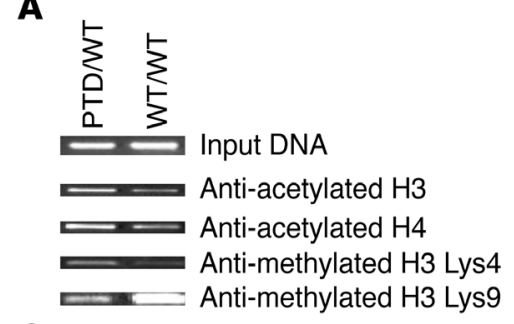

C

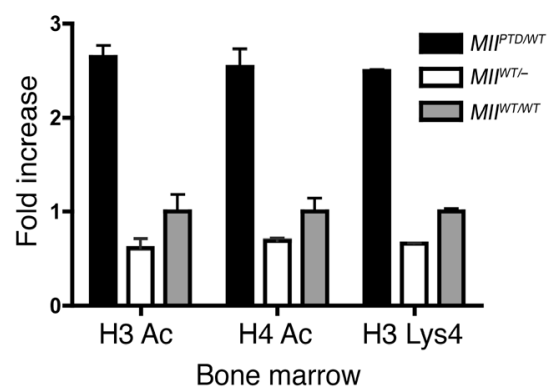

Figure 8

Assessment of MII target gene Hoxa9 acetylation and methylation of histones assessed by ChIP using (A) bone marrow cells and (B) splenocytes derived from MIIPTD/WT and MIIWT/WT mice. Results show an increase at the Hoxa9 promoter in histone $\mathrm{H} 3 / \mathrm{H} 4$ acetylation as well as histone H3 Lys4 methylation and a corresponding decrease in histone H3 Lys9 methylation in MIIPTD/WT bone marrow and spleen compared with the same MIIWT/WT tissues. Results are representative of 3 independent experiments. Similar results were obtained at the Hoxa7 promoter but were not seen at the Hoxa10 promoter (not shown). (C) BM cells and (D) splenocytes derived from $M I I^{P T D / W T}$, $M I I^{W T /}$, and $M I I^{W T} / W T$ mice. Absolute quantification by SYBR green real-time PCR shows a significant increase at the Hoxa9 promoter in histone $\mathrm{H} 3 / \mathrm{H} 4$ acetylation as well as histone H3 Lys4 methylation in MIIPTD/WT BM and spleen compared with the same MIIWT- and MIIWT/WT tissues ( $n=3$ mice per genotype; $P<0.05)$. There was no significant difference between MIIWT/- and MIIWT/WT tissues.

known as histone acetyltransferases, such as cAMP response element-binding protein-binding protein (CBP), are responsible for histone acetylation and associate with MLL in a complex via its $C$ terminal transactivation domain, which is uniquely preserved in the MLL PTD (34). There are genetic data showing evolutionarily conserved association of the MLL homolog Trx with Drosophila CBP in the fly, thus demonstrating the importance of this protein interaction (35). Other complexes found within the MLL supercomplex function as either activators or repressors of transcription, some via acetylation $(7,8)$. Examination of the histone acetylation states at Hoxa7 and Hoxa9 promoters in our study showed overacetylation of histone $\mathrm{H} 3 / \mathrm{H} 4$ in spleen and $\mathrm{BM}$ of $M l^{P T D / W T}$ mice, explaining at least 1 mechanism by which the $M l l$ PTD is mediating HoxA overexpression. The specific complexes associating with the $M l l$ PTD that are responsible for this observation and the direct or indirect mechanism by which this is accomplished have yet to be elucidated.

Like the transactivation domain, the SET domain is also retained in the C terminus of the MLL PTD, again in contrast to all other $M L L$ gene fusions found in AML. The SET domain of MLL has histone H3 Lys4 methyltransferase activity essential for maintaining Hox expression $(7,8)$. We hypothesized that histone H3 Lys4 methylation might also contribute to upregulation of these HoxA genes. Indeed, ChIP experiments revealed a correlation between overexpression of both Hoxa7 and Hoxa9 transcript with increases of histone H3 Lys4 methylation at these promoters in Mll $l^{P T D} / W T$ tis- unknown but must be directly or indirectly related to the PTD itself. Several possible mechanisms are now conceivable with the recent evidence that WT MLL directly binds to the promoter of these HoxA target genes, together with its supercomplex of repressing and activating proteins $(7,8)$. The duplication of the DNA-binding AT-hook DNA-binding domain within the Mll PTD may have conferred sustained and therefore excessive binding and increased activation at the relevant Hoxa7 and Hoxa9 promoters or could in some way enhance the recruitment of the Mll PTD and its supercomplex to its target genes (37). Alternatively, the associated supercomplex of activating and repressing components may be altered by the PTD in a way that changes a critical balance between these various complexes, excessively favoring target gene activation. It is conceivable that the binding of the Mll PTD to the promoter of these HoxA genes may interfere with the recruitment of the repression machinery. Careful biochemical characterization of these regulatory regions within the promoters of downstream targets of Mll will provide further insights into the dynamic regulation of these gene products in vivo.

Retroviral overexpression of Hox genes, including HoxA members, has been associated with massive deregulation of hematopoiesis and leukemia (27). Despite the sometimes substantial overexpression of the same HoxA genes observed in our $M l l^{P T D / W T}$ mice, they displayed no signs of malignant transformation at 2 years of age. The increase in $M l l^{P T D / W T}$ progenitor cell number and proliferation during $\mathrm{CFU}$ and liquid culture assays, respectively, suggests that HoxA overexpression exerts 
Table 4

Assessment of MIIPTDWT and MIIWT/- phenotypes compared with MIINTWT genotype

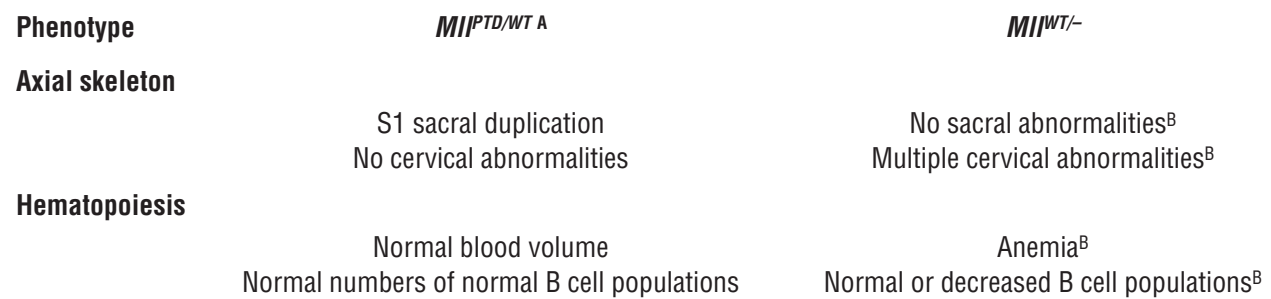

\section{Function}

Significantly increased $\mathrm{H} 3 / \mathrm{H} 4$ acetylation and H3 Lys4 methylation at Hoxa7 and Hoxa9 promoters ${ }^{\mathrm{C}}$
Normal or decreased ${ }^{A, C}$

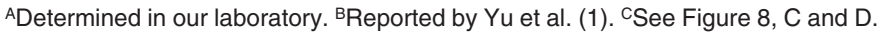

Development of the Mll ${ }^{P T D / W T}$ mouse. A P1 genomic clone containing the murine Mll genomic fragment was obtained from Incyte (formerly Genome Systems). This was subcloned as 2 fragments (F1 and F2) into pBluescript II as BamHI fragments. The BamHI/SmeI fragment from $\mathrm{F} 2$ was then subcloned into the BamHI and SmeI sites of ploxPneo1. $\mathrm{F} 1$ was then subcloned into the BamHI site of ploxPneo containing F2. This generated a targeting vector with ploxPneo 1 as the vector backbone and genomic $M l l$ sequences

its effect on immature cells of the hematopoietic compartment but by itself is insufficient to cause leukemic transformation. Overexpression of Hoxa7 or Hoxa9 rapidly induces AML in mice but requires forced coexpression of the Hox cofactor Meis1 (16). $M l l^{P T D / W T}$ cells overexpressing Hoxa7 and Hoxa9 genes in our study did not display any increase in Meis1 transcript. Further, AML patients harboring the MLL PTD also undergo epigenetic silencing of their WT MLL allele in their leukemic blasts that contributes to enhanced cell survival (13). Our Mll PTD mice express the WT $M l l$ allele, presumably because this later event requires cooperation with other alterations. Finally, the $M L L$ PTD has been shown to occur simultaneously with tyrosine kinase mutations in AML, such as the FLT3 ITD (38) and Rhe-PDGFR $\alpha$ fusion (39), neither of which has been detected in our $M l l^{P T D / W T}$ mice. Thus, the failure of our mice to develop frank leukemia is likely related to the absence of additional molecular aberrations that are critical for malignant transformation to occur in the presence of the Mll PTD.

While it would seem most logical that we are observing the earliest events in a continuum of how the Mll PTD contributes to the leukemic phenotype, it must also be considered that some or all of the abnormalities we have observed may not be related to the leukemogenic process as it occurs in patients with AML and the MLL PTD. For example, while the excessive production of erythroid and megakaryocytic progenitor populations is consistent with the specific role of Hoxa10 in these lineages (28) and with Hoxa10 overexpression found in the $M I l^{P T D / W T}$ mice, AML patients with the MLL PTD rarely have blasts of erythroid or megakaryocyte lineage. Clearly, additional work in combination with mice harboring other genetic defects, as described above, will need to be undertaken in order to shed light on the role of the Mll PTD in the genesis of leukemia.

In summary, we have engineered a mouse with the Mll PTD driven off of its endogenous promoter. We provide what we believe to be the first in vivo evidence for a mechanism by which an $M l l$ gene fusion (in this case, a self fusion) strongly associated with AML leads to aberrant target gene activation, i.e., increased histone $\mathrm{H} 3$ / $\mathrm{H} 4$ acetylation and histone H3 Lys4 methylation.

\section{Methods}

All primers and primer/probe sets used, along with conditions of amplification can be found in Supplemental Tables 1 and 2 . spanning intron 4 through intron 11 (Figure 1). All animal research was reviewed and approved by the Institutional Laboratory Animal Care and Use Committee at the Ohio State University.

Approximately $60 \mu \mathrm{g}$ of the targeting vector was linearized within intron 4 of $M l l$ using the unique SfiI restriction site and introduced into $3 \times 10^{7} \mathrm{R} 1 \mathrm{ES}$ cells. A plasmid vector expressing Cre recombinase from a CMV promoter ( $\mathrm{pCre}$ ) that conferred puromycin resistance was transiently transfected into $\mathrm{C} 186 \mathrm{MlPTD/WT}$ ES cells by electroporation. Cells were cultured overnight and then transferred to a selective media containing 1 $\mu \mathrm{g} / \mathrm{ml}$ of puromycin, grown further, and subcloned. Following transient transfection, selection, and expansion, DNA-PCR was performed using an upstream primer from intron 11 and a downstream primer from the ploxPneo vector. This amplified a $0.9-\mathrm{kb}$ band, suggesting that the pgkneo cassette had been removed, and this was confirmed by sequencing. Next, 2 independently targeted ES cell clones were selected for generating chimeric mice. After verifying that these clones were karyotypically normal and free of mycoplasma contamination, 15 ES cells from each clone were injected into 3.5-day-old blastocysts from pregnant C57BL/ 6 female mice (The Jackson Laboratory). Next, the chimeric embryos were transferred into pseudopregnant recipient Swiss-Webster female mice. Micromanipulations, blastocyst transfers, and generation of chimeric mice were performed at the Transgenic Shared Resource of The Ohio State University Comprehensive Cancer Center. Backcrossing to a pure C57BL/6 background was carried out in 5 generations using marker-assisted breeding (speed congenics) (The Jackson Laboratory).

Southern blot analysis. High molecular weight DNA extracted from cells was digested with Sbfl and NdeI (New England Biolabs Inc.), and Southern blotting was performed according to previously described techniques (40). DNA was transferred to Hybond $\mathrm{N}+$ nitrocellulose membrane (Amersham Biosciences) and hybridized to a cDNA fragment spanning the $M l l$ intron 4-exon 5 junction. The predicted fragment size for the Mll PTD allele was $40 \mathrm{~kb}$ while the predicted fragment size for the WT Mll allele was $18 \mathrm{~kb}$.

Genotyping. DNA was prepared from tail biopsies of adult and newborn mice using standard phenol/chloroform extraction. DNA PCR was performed using a forward primer located within intron 6 and a reverse primer located within the targeting vector backbone that amplifies a 900-bp product. Total RNA was extracted from tissues using the RNeasy Mini Kit (QIAGEN), and RT-PCR was performed using MMLV Reverse Transcriptase (Invitrogen) with a forward primer located within exon 11 and a reverse primer located within exon 6 , which resulted in the generation of a 335-bp PCR product. 
CFU progenitor assays and liquid stem cell cultures. Splenocytes were isolated from $M l l^{P T D / W T}, M l l^{W T / W T}$, and $M l l^{a f 9 / W T}$ mice (generously provided by T. Rabbitts, Medical Research Council Laboratory of Molecular Biology, Cambridge, United Kingdom, via J. Kersey, Cancer Center, University of Minnesota, Minneapolis, Minnesota, USA). Single-cell suspensions were prepared in Iscove's Modified Dulbecco's Medium (Invitrogen) containing $2 \%$ of FBS (HyClone) from $n=6-8$ mice per genotype. Cells were then plated at a density of $2 \times 10^{4}$ cells/dish in M3434 methylcellulose (StemCell Technologies) to assess splenic progenitor populations. All colony assays were performed according to the manufacturer's protocol (StemCell Technologies). Secondary replating assays were enumerated and performed on day 14 of primary cultures. Cells were replated at a density of $10^{4}$ cells/dish in M3434 methylcellulose (StemCell Technologies). Secondary cultures were also performed in liquid medium RPMI 1640 with GlutaMAX (Invitrogen) plus $100 \mathrm{ng} / \mathrm{ml}$ of stem cell factor, $10 \mathrm{ng} / \mathrm{ml}$ of mouse IL-3, and $20 \mathrm{ng} / \mathrm{ml}$ of human IL-6 (StemCell Technologies) and seeded simultaneously from primary CFU progenitor assays and in parallel with secondary replating assays at a density of $10^{4}$ cells $/ \mathrm{ml}$.

ChIP. ChIP assays were performed using the Chromatin Immunoprecipitation Assay Kit (Upstate USA Inc.). Approximately $3 \times 10^{6}$ cells were used per assay, and approximately $1 \%$ of the extracted chromatin solution was saved for use as input DNA. Immunoprecipitation with the following antibodies was performed: anti-acetyl-Histone $\mathrm{H} 4$, anti-acetyl-Histone $\mathrm{H} 3$, anti-dimethyl-Histone $\mathrm{H} 3$ Lys4, and antidimethyl-Histone H3 Lys9 (Upstate USA Inc.). The DNA was extracted using phenol-chloroform, precipitated with ethanol, and dissolved in water. Immunoprecipitations were analyzed by nested PCR using GeneAmp PCR System 9700 (Applied Biosystems). The cycle number and the amount of template were optimized to ensure that results were within the linear range of amplification. The PCR products were size fractionated through $1.0 \%$ agarose/ethidium bromide gels.

Quantification of histone acetylation and methylation at Hoxa7 and Hoxa9 promoters in $M l l^{P T D / W T}$, $M l l^{W T /-}$ (generously provided by the late S. Korsmeyer), and $M l l^{W T / W T}$ DNA extracted from spleen and BM cells was undertaken using real-time quantitative PCR with SYBR green incorporation (Applied Biosystems). Primers were designed and optimized to amplify genomic sequences in the Hoxa 7 and Hoxa9 promoters (Supplemental Tables 1 and 2). Each reaction used $2 \mu$ l of DNA template and 12.5 $\mu$ l SYBR Green PCR Master Mix (Applied Biosystems) and was normalized to input DNA. The specificity of PCR products was analyzed by addition of a melting curve cycle, which consisted of 1 cycle each of $60^{\circ} \mathrm{C}$ for 15 seconds, $95^{\circ} \mathrm{C}$ for 20 seconds, and $60^{\circ} \mathrm{C}$ for 15 seconds, followed by analysis on Dissociation Curve Analysis software, version 1.0 (Applied Biosystems).

Comparative real-time RT-PCR. Total RNA was extracted from mouse BM, spleen, and peripheral blood samples and cDNA prepared as previously described (41). Primer and probe sets to detect murine Hoxa7, Hoxa9, and Hoxa10 transcripts were designed using Primer Express (Applied Biosystems). Real-time RT-PCR efficiency was optimized for each primer/probe set using established guidelines (User Bulletin, Applied Biosystems; http://www.appliedbiosystems.com/support/apptech/). Comparative real-time RT-PCR was performed following the manufacturer's suggested procedures and using the ABI Prism 7700 Sequence Detection System (Applied Biosystems). To normalize for RNA content, the $\mathrm{r} 18 \mathrm{~s}$ primer/probe set was included in multiplex reactions. Data were analyzed using SDS version 1.7a software (Applied Biosystems) and reported as $2^{-\Delta \Delta C t}$, which represents the fold differences when normalized to an internal control (r18s) and then compared with MllWT/WT sibling control samples analyzed on the same 96-well plate. For cell-sorting real-time RT-PCR experiments, whole BM cells were harvested and stained with antibodies to Ter119, CD71, Gr-1, CD11b, B220, and CD3. A minimum of $1 \times 10^{5}$ cells positive for each lineage marker was isolated using a FACSVantage (BD). Equivalent numbers of cells positive for each marker from $M l l^{W T / W T}$ and $M l l^{P T D / W T}$ were used to quantify HoxA gene expression. Real-time RT-PCR was then performed as described above. We used our previously published methodology to quantify absolute levels of Mll PTD and Mll WT transcripts present in BM, spleen, and blood of Mll ${ }^{P T D / W T}$ mice by real-time RT-PCR (13). Further information on primers and probes is provided in Supplemental Methods.

Flow cytometric analysis for lineage and subset determination, proliferation, and apoptosis. Cell lineages and hematopoietic subsets within the BM, spleen, and peripheral blood were assessed using lineage-specific antibodies as previously described $(15,41)$. Immunofluorescent analysis of antigen expression was performed using a FACSCalibur (BD) and CellQuest software (version 3.3; BD). For quantitative assessment of cellular proliferation, BrdU incorporation was measured according to the manufacturer's protocol (BD Biosciences - Pharmingen). To assess differences in apoptosis, annexin $\mathrm{V}$ and PI staining were used and performed according to manufacturer's protocol (BD Biosciences - Pharmingen).

Whole mount in situ. Whole mount in situ was performed on E12.5 embryos as previously described (1), using digoxygenin-labeled riboprobes generated according to manufacturer's protocols (Roche Diagnostics). Primer pairs used to generate both sense and anti-sense probes first standardized on WT embryos can be obtained in Supplemental Tables 1 and 2.

Skeletal analysis. Intact skeletons were prepared using sex-matched $M l l^{P T D / W T}$ and $M l l^{W T / W T}$ mice 20 weeks of age using previously described methods (42). The microCT images were performed as follows: the sacral vertebrae specimens were mounted in $50-\mathrm{ml}$ plastic test tubes filled with glycerol and imaged at $32 \mathrm{kV}, 1 \mathrm{~mA}$, and 1-second exposure with image intensifier operating in 7 -inch mode. The spatial resolution of the images is $60 \mu \mathrm{m}$.

Statistics. A Wilcoxon's signed-rank test was used to compare differences in CFU growth between the $M l l^{W T / W T}$ and $M l l^{P T D / W T}$ splenocytes. The Hoxa7, Hoxa9, and Hoxa10 expression levels for each tissue were compared between $M l l^{W T / W T}$ and $M l l^{P T D / W T}$ mice using a 2-sample 2-tailed Student's $t$ test.

\section{Acknowledgments}

This work was funded in part by the Lady Tata Memorial Trust Award (to A.M. Dorrance) and National Cancer Institute grant funding (R01 CA89341 to M.A. Caligiuri, K01 CA96887 to S.P. Whitman, and K08 CA089317 to L.J. Rush). The microCT images were provided by Kim Powell at the Cleveland Clinic Musculoskeletal Imaging Core, funded in part by a National Institute of Arthritis and Musculoskeletal and Skin Diseases (NIAMS) Core Center grant (P30 AR050953-01). We thank Jay Hess for kindly providing the Meis1 primer/probe set for quantification of Meis1 transcript, Terry Rabbitts and John Kersey for providing the Mllaf $9 / W T$ mice, the late Stanley Korsmeyer for providing the $M l l^{W T} /-$ mice, and the staff of the Transgenic Animal, DNA, and Mouse Phenotyping Shared Resources at The Ohio State University Comprehensive Cancer Center for their assistance.

Received for publication May 3, 2005, and accepted in revised form July 25, 2006.

Address correspondence to: Michael A. Caligiuri, The Ohio State University, Comprehensive Cancer Center, 320 West 10th Avenue, Columbus, Ohio 43220, USA. Phone: (614) 293-7521; Fax: (614) 293-7522; E-mail: michael.caligiuri@osumc.edu. 
1. Yu, B.D., Hess, J.L., Horning, S.E., Brown, G.A., and Korsmeyer, S.J. 1995. Altered Hox expression and segmental identity in Mll-mutant mice. Nature. 378:505-508

2. Zeleznik-Le, N.J., Harden, A.M., and Rowley, J.D. 1994. 11q23 translocations split the "AT hook" cruciform DNA binding region and the transcriptional repression domain from the activation domain of the mixed-lineage leukemia (MLL) gene. Proc. Natl. Acad. Sci. U. S. A. 91:10610-10614.

3. Caligiuri, M.A., et al. 1994. Molecular rearrangement of the ALL-1 gene in acute myeloid leukemia without cytogenetic evidence of 11q23 chromosomal translocations. Cancer Res. 54:370-373.

4. Caligiuri, M., et al. 1998. Rearrangement of $A L L 1$ $(M L L)$ in acute myeloid leukemia with normal cytogenetics. Cancer Res. 58:55-59.

5. Dohner, K., et al. 2002. Prognostic significance of partial tandem duplications of the MLL gene in adult patients 16 to 60 years old with acute myeloid leukemia and normal cytogenetics: a study of the Acute Myeloid Leukemia Study Group Ulm. J. Clin. Oncol. 20:3254-3261.

6. Golub, T.R., et al. 1999. Molecular classification of cancer: class discovery and class prediction by gene expression monitoring. Science. 286:531-537.

7. Nakamura, T., et al. 2002. ALL-1 is a histone methyltransferase that assembles a supercomplex of proteins involved in transcriptional regulation. Mol. Cell. 10:1119-1128.

8. Milne, T.A., et al. 2002. MLL targets SET domain methyltransferase activity to Hox gene promoters. Mol. Cell. 10:1107-1117.

9. Milne, T.A., Martin, M.E., Brock, H.W., Slany, R.K., and Hess, J.L. 2005. Leukemogenic MLL fusion proteins bind across a broad region of the Hox a9 locus, promoting transcription and multiple histone modifications. Cancer Res. 65:11367-11374.

10. Kumar, A.R., et al. 2004. Hoxa9 influences the phenotype but not the incidence of Mll-AF9 fusion gene leukemia. Blood. 103:1823-1828.

11. Dobson, C.L., et al. 1999. The mll-AF9 gene fusion in mice controls myeloproliferation and specifies acute myeloid leukaemogenesis. EMBO J. 18:3564-3574.

12. Hsieh, J.J., Cheng, E.H., and Korsmeyer, S.J. 2003. Taspase1: a threonine aspartase required for cleavage of MLL and proper HOX gene expression. Cell. 115:293-303.

13. Whitman, S.P., et al. 2005. The MLL partial tandem duplication: evidence for recessive gain-of-function in acute myeloid leukemia identifies a novel patient subgroup for molecular-targeted therapy.
Blood. 106:345-352.

14. Caligiuri, M.A., et al. 1997. The partial tandem duplication of ALL1 in acute myeloid leukemia with normal cytogenetics or trisomy 11 is restricted to 1 chromosome. Proc. Natl. Acad. Sci. U. S. A. 94:3899-3902.

15. Na Nakorn, T., Traver, D., Weissman, I.L., and Akashi, K. 2002. Myeloerythroid-restricted progenitors are sufficient to confer radioprotection and provide the majority of day 8 CFU-S. J. Clin. Invest. 109:1579-1585. doi:10.1172/JCI200215272.

16. Nakamura, T., Largaespada, D.A., Shaughnessy, J.D., Jr., Jenkins, N.A., and Copeland, N.G. 1996. Cooperative activation of Hoxa and Pbx1-related genes in murine myeloid leukaemias. Nat. Genet. 12:149-153.

17. Moskow, J.J., Bullrich, F., Huebner, K., Daar, I.O., and Buchberg, A.M. 1995. Meis1, a PBX1-related homeobox gene involved in myeloid leukemia in BXH-2 mice. Mol. Cell. Biol. 15:5434-5443.

18. Kroon, E., et al. 1998. Hoxa9 transforms primary bone marrow cells through specific collaboration with Meis1a but not Pbx1b. EMBOJ. 17:3714-3725.

19. Duboule, D and Dolle, P. 1989. The structural and functional organization of the murine HOX gene family resembles that of Drosophila homeotic genes. EMBO J. 8:1497-1505.

20. Kessel, M., Balling, R., and Gruss, P. 1990. Variations of cervical vertebrae after expression of a Hox-1.1 transgene in mice. Cell. 61:301-308.

21. Morgan, B.A., Izpisua-Belmonte, J.C., Duboule, D., and Tabin, C.J. 1992. Targeted misexpression of Hox-4.6 in the avian limb bud causes apparent homeotic transformations. Nature. 358:236-239.

22. Lufkin, T., et al. 1992. Homeotic transformation of the occipital bones of the skull by ectopic expression of a homeobox gene. Nature. 359:835-841.

23. Fromental-Ramain, C., et al. 1996. Specific and redundant functions of the paralogous Hoxa-9 and Hoxd-9 genes in forelimb and axial skeleton patterning. Development. 122:461-472.

24. Drabkin, H.A., et al. 2002. Quantitative HOX expression in chromosomally defined subsets of acute myelogenous leukemia. Lenkemia. 16:186-195.

25. Rozovskaia, T., et al. 2001. Upregulation of Meis1 and HoxA9 in acute lymphocytic leukemias with the $\mathrm{t}(4: 11)$ abnormality. Oncogene. 20:874-878.

26. Fujino, T., et al. 2001. Inhibition of myeloid differentiation by Hoxa9, Hoxb8, and Meis homeobox genes. Exp. Hematol. 29:856-863.

27. Buske, C., and Humphries, R.K. 2000. Homeobox genes in leukemogenesis. Int. J. Hematol. 71:301-308.

28. Buske, C., et al. 2001. Overexpression of HOXA10 perturbs human lymphomyelopoiesis in vitro and in vivo. Blood. 97:2286-2292.

29. Kawagoe, H., Humphries, R.K., Blair, A., Sutherland, H.J., and Hogge, D.E. 1999. Expression of HOX genes, HOX cofactors, and MLL in phenotypically and functionally defined subpopulations of leukemic and normal human hematopoietic cells. Leukemia. 13:687-698.

30. Armstrong, S.A., et al. 2002. MLL translocations specify a distinct gene expression profile that distinguishes a unique leukemia. Nat. Genet. 30:41-47.

31. Jenuwein, T., and Allis, C.D. 2001. Translating the histone code. Science. 293:1074-1080.

32. Zhang, Y., and Reinberg, D. 2001. Transcription regulation by histone methylation: interplay between different covalent modifications of the core histone tails. Genes Dev. 15:2343-2360.

33. Lachner, M., and Jenuwein, T. 2002. The many faces of histone lysine methylation. Curr. Opin. Cell Biol. 14:286-298.

34. Ernst, P., Wang, J., Huang, M., Goodman, R.H., and Korsmeyer, S.J. 2001. MLL and CREB bind cooperatively to the nuclear coactivator CREB-binding protein. Mol. Cell. Biol. 21:2249-2258.

35. Petruk, S., et al. 2001. Trithorax and dCBP acting in a complex to maintain expression of a homeotic gene. Science. 294:1331-1334.

36. Milne, T.A., et al. 2005. MLL associates specifically with a subset of transcriptionally active target genes. Proc. Natl. Acad. Sci. U. S. A. 102:14765-14770.

37. Martin, M.E., et al. 2003. Dimerization of MLL fusion proteins immortalizes hematopoietic cells. Cancer Cell. 4:197-207.

38. Steudel, C., et al. 2003. Comparative analysis of MLL partial tandem duplication and FLT3 internal tandem duplication mutations in 956 adult patients with acute myeloid leukemia. Genes Chromosomes Cancer. 37:237-251.

39. Quentmeier, H., Reinhardt, J., Zaborski, M., and Drexler, H.G. 2003. MLL partial tandem duplications in acute leukemia cell lines. Lenkemia. 17:980-981.

40. Caligiuri, M.A., et al. 1996. Partial tandem duplication of ALL1 as a recurrent molecular defect in acute myeloid leukemia with trisomy 11. Cancer Res. 56:1418-1425.

41. Fehniger, T.A., et al. 2001. Fatal leukemia in interleukin 15 transgenic mice follows early expansions in natural killer and memory phenotype CD8(+) T cells. J. Exp. Med. 193:219-232.

42. McLeod, M.J. 1980. Differential staining of cartilage and bone in whole mouse fetuses by alcian blue and alizarin red S. Teratology. 22:299-301. 